\title{
Männlicher Schweiß wirkt gegen Schmerzen
}

Laborexperimente mit Nagetieren werden normalerweise unbeachtet des Geschlechts des Versuchspersonals durchgeführt. Dass das Geschlecht der Experimentatoren einen Einfluss auf Versuchsergebnisse haben bzw. diese sogar signifikant verändern kann, haben kanadische Forscher von der McGill University in Montreal herausgefunden.

Die Anwesenheit bestimmter Substanzen, die sich im männlichen Schweiß befinden, löst bei Nagetieren stressinduzierte Analgesie (SIA) aus. Zu diesem Schluss kamen die Wissenschaftler um Robert E. Sorge, die die Schmerzempfindung von Mäusen und Ratten in Abhängigkeit des Geschlechts des Versuchspersonals untersuchten.

Die Wissenschaftler führten Experimente durch, in denen sie Mäusen und Ratten mittels bilateraler Injektion von Zymosan A in

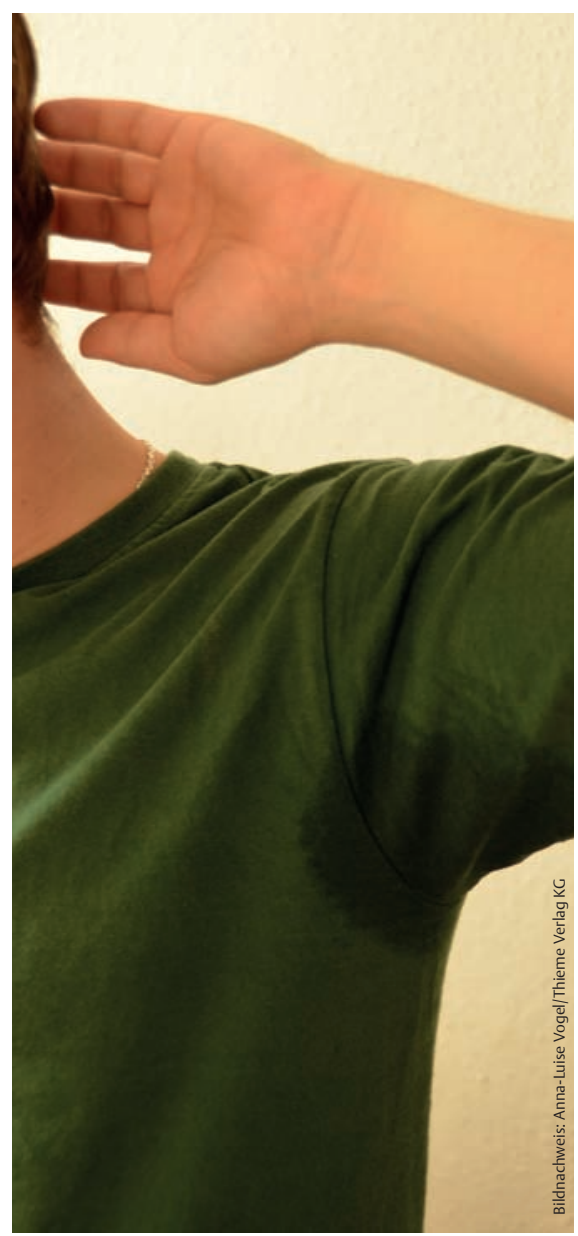

die Fußgelenke schmerzhafte Entzündungen zufügten und sie danach beobachteten, um die Schmerzempfindung der Tiere nach der sog. „Mouse-Grimace-Skala“ zu quantifizieren. In Anwesenheit von männlichen Beobachtern zeigten die Tiere signifikant weniger Schmerzempfindung als bei weiblichen Beobachtern oder ohne Beobachter. Dabei spielte das Geschlecht der Person, die die Tiere versorgte oder das Zymosan verabreichte, keine Rolle. Dieser „männliche Beobachter-Effekt" trat bei Versuchstieren beider Geschlechter auf und ging nach 3060 min wieder vollständig zurück. Der gleiche Effekt trat auch auf, wenn statt der Beobachter nur T-Shirts, die in der Nacht zuvor von weiblichem bzw. männlichem Personal getragen worden waren, platziert wurden, was zu der Annahme führte, dass das Phänomen olfaktorischer Natur ist.

In einem weiteren Versuch untersuchten die Forscher nicht nur den Gesichtsausdruck, sondern auch das durch den Schmerz verursachte Verhalten, wie z. B. Lecken und Beißen an der Injektionsstelle. Hierfür injizierten sie den Mäusen 1\%- und 5\%-iges Formalin und stellten fest, dass bei der höheren Konzentration olfaktorische Stimuli alleine nicht mehr ausreichten, sondern auch wiederholte visuelle Stimuli notwendig waren, um den Effekt zu erzielen.

Um die für den Effekt verantwortlichen Stoffe zu identifizieren, untersuchten die Forscher die Wirkungen einzelner Stoffe, die im männlichen Achselschweiß vermutlich stärker vorhanden sind als im weiblichen. Sie fanden 3 Substanzen (3M2H, Androstenon, Androstadienon), die das Schmerzempfinden der Mäuse signifikant beeinflussten. Da Chemosignale in allen Säugerspezies strukturell gleich sind, testeten sie, ob der Geruch unbekannter männlicher Mäuse, männlicher Meerschweinchen und männlicher Fressfeinde (Hund, Katze, Ratte) bei Mäusen auch Analgesie hervorruft. Das war der Fall. Handelte es sich dabei allerdings um den Geruch von Käfiggenossen, trat der Effekt nicht auf. Es gab nun 2 mögliche Erklärungen für die beobachtete Analgesie:
1. Die Mäuse unterdrücken bewusst äußerliche Anzeichen von Schmerzempfindung.

2. Die Stimuli verursachen stressinduzierte Analgesie (SIA).

SIA ist eine angeborene Reaktion auf Stress, bei der die Schmerzverarbeitung im Rückenmark unterdrückt wird. Und in der Tat konnten die Wissenschaftler Anzeichen von Stress nachweisen, der alleine durch die Wahrnehmung männlichen Geruchs ausgelöst wird, wie z.B. der Anstieg des Corticosteronspiegels im Blut, die Entwicklung von Hyperthermie und die mit SIA einhergehende herabgesetzte Expression eines Immediate-Early-Gens in schmerzverarbeitenden Neuronen.

Aufgrund ihrer Ergebnisse vermuteten die Wissenschaftler, dass die Basissensitivität von Versuchstieren bei präklinischen Schmerzversuchen durch den Experimentator beeinflusst werden kann. Sie sahen sich deshalb veranlasst, archivierte Versuchsdaten zu re-analysieren und fanden, dass die Schmerzsensitivität der Mäuse in Schmerzassays bei männlichen Experimentatoren signifikant niedriger war als bei weiblichen, was auf einen möglichen analgetischen Effekt hinweist.

Fazit Die Ergebnisse deuten darauf hin, dass ein Cocktail von Chemosignalen aus männlichen Körpersekreten Stress in Nagetieren hervorruft, der zu SIA führt. Dieser „männliche Experimentator-Effekt“ könnte die Ergebnisse bisheriger Tierforschung infrage stellen. In Zukunft sollte daher das Geschlecht der Experimentatoren in der Standardlaborpraxis von Versuchen, die möglicherweise durch Stress beeinflusst werden könnten, berücksichtigt werden.

\section{Dr. rer.nat. Christine Seelmann, Düsseldorf}

Sorge RE, Martin LJ, Isbester KA et al. Olfactory exposure to males, including men, causes stress and related analgesia in rodents. Nat Methods 2014; 11: 629-632 